\title{
Estudio del régimen de pérdidas dieléctricas constantes en conductores iónicos con estructura de tipo pirocloro
}

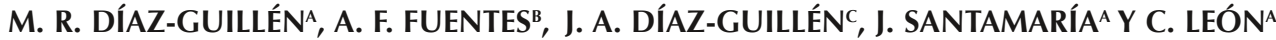 \\ ${ }^{a}$ GFMC, Departamento de Física Aplicada III, Facultad de Física, Universidad Complutense de Madrid, Campus Moncloa, 28040 Madrid, España \\ ${ }^{\text {b } C i n v e s t a v-S a l t i l l o, ~ A p a r t a d o ~ P o s t a l ~ 663, ~} 25000$ Saltillo, Coahuila, México \\ c División de Estudios de Posgrado e Investigación, Instituto Tecnológico de Saltillo, V. Carranza 2400, 25280 Saltillo, Coahuila, México
}

\begin{abstract}
Se presentan estudios de conductividad $a c$ en conductores iónicos con estructura de tipo pirocloro de fórmula $\mathrm{Gd}_{2}\left(\mathrm{Zr}_{\mathrm{y}} \mathrm{Ti}_{1-\mathrm{y}}\right)_{2} \mathrm{O}_{7}$ a temperaturas entre -20 y $250^{\circ} \mathrm{C}$ y en un rango de frecuencias de $1 \mathrm{~Hz}$ a $3 \mathrm{MHz}$. Dicho estudio revela que existe un transición de una región de dependencia potencial a una región de dependencia lineal (conocida como régimen de pérdidas dieléctricas constantes o NCL) de la conductividad con la frecuencia en un amplio rango de temperaturas. Esta transición resulta estar térmicamente activada y su energía de activación $E_{N C L}$ es mucho menor que la energía de activación $E_{d c}$ para el proceso de conducción de largo alcance. Los valores de $E_{N C I^{\prime}}$ a diferencia de $E_{d c^{\prime}}$ son prácticamente independientes de la composición y por tanto de la concentración de vacancias de oxígeno. Por otro lado y para cada composición, los valores de $E_{N C L}=0.67 \pm 0.04$ eV son muy similares a los calculados para la energía que los iones necesitan superar para salir de sus pozos de potencial, $E_{a}=0.69 \pm 0.05 \mathrm{eV}$. Los resultados obtenidos soportan la teoría que confirma que el régimen NCL es consecuencia de las vibraciones locales reflejadas en el desplazamiento cuadrático medio de los iones cuando estos permanecen confinados en su pozo de potencial.
\end{abstract}

\section{Palabras clave: Propiedades eléctricas/dieléctricas. Conductividad. Conductores iónicos.}

\section{Study of the nearly constant dielectric loss regime in ionic conductors with pyrochlore-like structure}

We report on ac conductivity measurement of oxide ion conductors with composition $\mathrm{Gd}_{2}\left(\mathrm{Zr}_{\mathrm{v}} \mathrm{Ti}_{1-\mathrm{y}}\right)_{2} \mathrm{O}_{7}$ and a pyrochloretype structure, at temperatures between -20 and $250^{\circ} \mathrm{C}$ and in the frequency range from $1 \mathrm{~Hz}$ to $3 \mathrm{MHz}$ by using impedance spectroscopy. Results show that a crossover from a power law dependence to a linear frequency dependence (or nearly constant loss behavior) in the ac conductivity can be clearly observed in a wide temperature range. This crossover is found to be thermally activated, and its activation energy $E_{\mathrm{NCL}}$ to be much lower than the activation energy $E_{d c}$ for the dc conductivity. We also found that the values of $E_{\mathrm{NCL}}$ are almost independent of composition, and therefore of the concentration of mobile oxygen vacancies, unlike those of $E_{d c}$. Moreover, for each composition, the values of $E_{\mathrm{NCL}}=0.67 \pm 0.04 \mathrm{eV}$ are very similar to those estimated for the energy barrier for the ions to leave their cages, $E_{a}=0.69 \pm 0.05 \mathrm{eV}$. These results support that the nearly constant loss behavior, ubiquitous in ionic conductors, is originated from caged ion dynamics.

Keywords: Electric/dielectric properties. Conductivity. Ionic conductors.

\section{INTRODUCCIÓN}

Un comportamiento universal recientemente estudiado sobre los conductores iónicos es el conocido como régimen de pérdidas dieléctricas constantes o NCL (Nearly Constant Loss), que se presenta en este tipo de materiales a temperaturas lo suficientemente bajas o altas frecuencias y el cual consiste en una dependencia lineal de la conductividad ac con la frecuencia y está descrito por $(1,2)$ :

$\sigma_{\mathrm{NCL}}^{\prime}(\omega) \approx A \varepsilon_{0} \omega \quad[1]$

donde $A$ es una constante que depende ligeramente de la temperatura y $\varepsilon_{0}$ corresponde a la permitividad en el vacío $(3,4)$. El término "régimen de pérdidas dieléctricas constantes" se debe a que las pérdidas dieléctricas presentan una dependencia prácticamente independiente con la frecuencia $\varepsilon_{N C L}{ }^{\prime \prime}(\omega)=\sigma^{\prime}{ }_{N C L}(\omega) / \omega \varepsilon_{0} \approx A$. A pesar de que los materiales conductores de iones han despertado un gran interés en la comunidad científica en las últimas décadas, la mayor parte de los estudios experimentales y teóricos se han enfocado principalmente en la comprensión de la dinámica de iones móviles a altas temperaturas, cuando los iones móviles contribuyen a un transporte de carga de largo alcance y que se caracteriza por el comportamiento independiente de la conductividad $d c$ con la frecuencia y en donde el NCL normalmente no se hace evidente en los datos experimentales. De hecho, hasta hace poco tiempo, los datos de conductividad que incluían el régimen NCL eran bastante escasos en la literatura (5-9). No fue sino hasta el año 1999 cuando Ngai estableció la correlación existente entre los elevados valores de conductividad $d c$ y los valores de la magnitud $A$ del régimen NCL encontrado en conductores iónicos $(4,10)$. Basado en este hecho y en algunas otras propiedades del NCL observadas experimentalmente en este tipo de materiales, Ngai propuso que el origen físico podría deberse al desplazamiento de los iones móviles durante su movimiento oscilatorio local a bajas temperaturas y/o altas frecuencias. 
Posteriormente se encontró evidencia de este comportamiento en dos diferentes conductores de iones litio, que concuerda con la propuesta de Ngai sobre el origen de la contribución del NCL a la conductividad ac (11-13). A partir, de medidas de la temperatura (frecuencia) de transición a la cual el régimen del NCL finaliza a diferentes frecuencias (temperaturas) fijas, fue posible determinar una energía de

a)

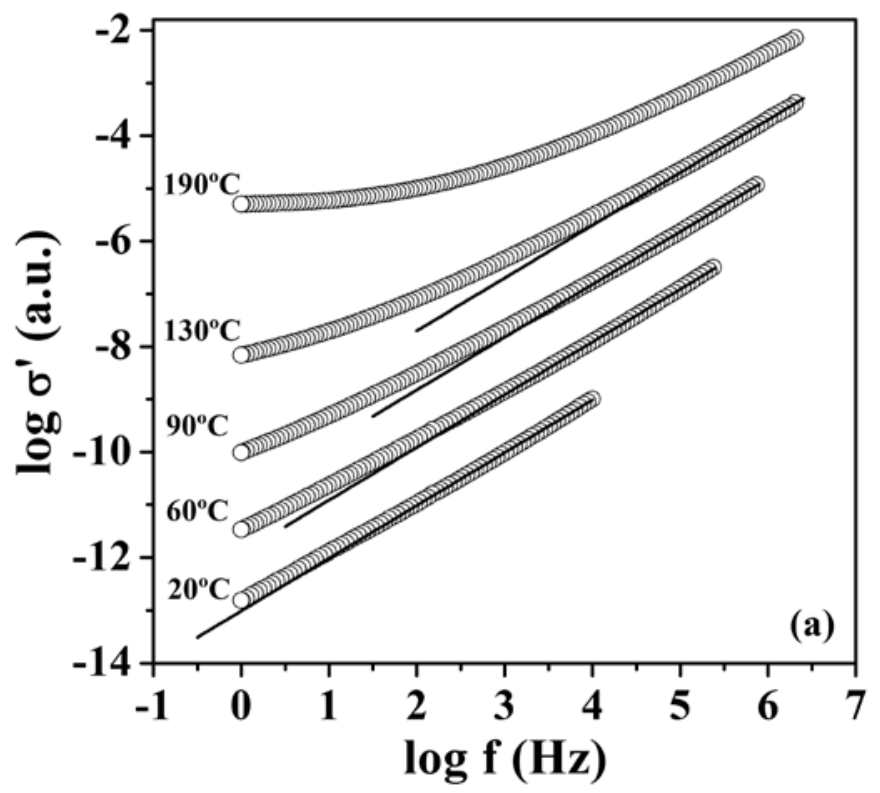

b)

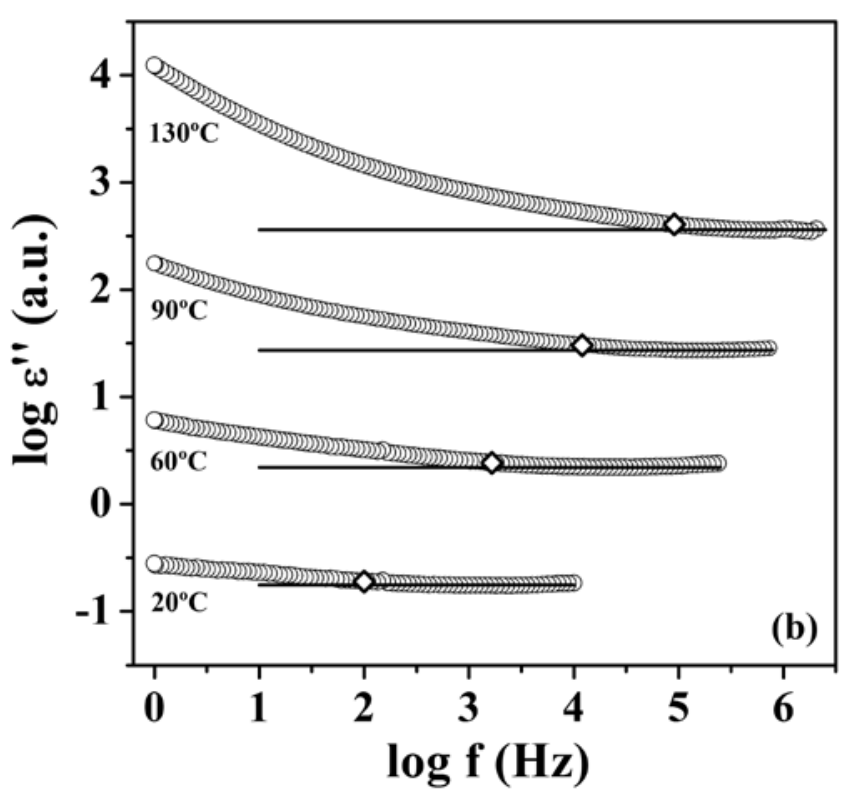

Figura 1. Dependencia de (a) parte real de la conductividad y (b) parte imaginaria de la permitividad en función de la frecuencia en escala doble logarítmica para la muestra $\mathrm{Gd}_{2} \mathrm{Zr}_{2} \mathrm{O}_{7}$ a distintas temperaturas. Las unidades de los datos de conductividad a $\mathrm{T}=20^{\circ} \mathrm{C}$ son $\mathrm{S} / \mathrm{cm}$ y por claridad, los demás datos han sido desplazados verticalmente 1, 2, 3 y 4 décadas para $\mathrm{T}=60,90,130$ y $190^{\circ} \mathrm{C}$ respectivamente. activación $E_{N C L}$ para dicha transición, la cual resultó ser mucho más baja que la energía de activación $E_{d c}$ para la conductividad dc que está determinada por la dinámica de iones móviles. De hecho, se encontró que la energía $E_{N C L}$ es similar a los valores calculados para la energía asociada a la barrera de potencial, $E_{d^{\prime}}$ que los iones oxígeno tienen que superar para saltar de un sitio a otro. Recientemente se han desarrollado diferentes modelos de conducción iónica en sólidos que han explicado dichos resultados experimentales basándose en las propiedades que presenta el NCL, en las que el pozo de potencial disminuye, lo cual resulta en una dependencia logarítmica del desplazamiento cuadrático medio con el tiempo y en consecuencia, en una dependencia lineal de la conductividad ac con la frecuencia $(14,15)$. Sin embargo, aun existe mucha controversia sobre el origen del NCL en conductores iónicos debido principalmente a la escasez de datos experimentales de conductividad ac que muestren su dependencia lineal con la frecuencia en un amplio rango de temperaturas y frecuencias, ya que el valor de las pérdidas dieléctricas, $A$, y por tanto el valor de $\tan \delta\left(\tan \delta=\varepsilon^{\prime \prime} / \varepsilon^{\prime}\right)$ son normalmente muy bajos en el NCL (16-20). En particular, no es fácil obtener suficiente resolución en las medidas del valor de la conductividad ac (pérdidas dieléctricas) para determinar de forma precisa la transición al régimen NCL mediante la variación de la temperatura y/o frecuencia. Dentro de este contexto, es de interés fundamental llevar a cabo estudios sistemáticos de la conductividad $a c$ a bajas temperaturas y/o altas frecuencias en diferentes materiales conductores iónicos que permitan obtener nuevos conocimientos acerca del origen y naturaleza de dicho régimen.

En este trabajo se presentan datos de conductividad $a c$ de varios circonatos conductores de iones oxígeno de fórmula general $\mathrm{Gd}_{2}\left(\mathrm{Zr}_{\mathrm{y}} \mathrm{Ti}_{1-\mathrm{y}}\right)_{2} \mathrm{O}_{7}$ los cuales presentan una estructura de tipo pirocloro con un alto grado de desorden estructural y que han demostrado ser materiales prometedores para sustituir a fluoritas tales como la circonia estabilizada con itria como electrolito sólido en celdas de combustible cerámicas $(21,22)$. Estructuralmente, los óxidos de este tipo se relacionan con la fluorita y como ésta, presentan conductividades considerables a elevadas temperaturas. Suelen ser cúbicos (grupo espacial Fd3m, 227), de fórmula general $\mathrm{A}_{2} \mathrm{~B}_{2} \mathrm{O}_{7} \mathrm{y}$ pueden describirse como una superestructura de la fluorita no estequiométrica. La diferencia fundamental entre ambas estructuras consiste en que mientras en las fluoritas las vacancias aniónicas están distribuidas al azar en la red cristalina, en los pirocloros están ordenadas aunque suelen desordenarse cuando se tratan a temperaturas o presiones suficientemente elevadas. Además, el desorden estructural en pirocloros se puede variar también de forma casi continua usando sistemas de soluciones sólidas por ejemplo del tipo $\mathrm{A}_{2}\left(\mathrm{Ti}_{1-y} \mathrm{Zr}_{y}\right)_{2} \mathrm{O}_{7}$, con estructuras de bajo a alto grado de desorden. Adicionalmente, también se ha encontrado que la existencia de una $u$ otra forma cristalina depende en gran medida del método usado para preparar las muestras en estudio así como de su historial térmico. Constituyen una familia de compuestos con interesantes propiedades eléctricas ya que dependiendo de su composición química, del orden/desorden existente en su arreglo atómico, y de la existencia de vacancias aniónicas, pueden mostrar conductividad electrónica, iónica o mixta. De hecho, en la solución sólida $\mathrm{Gd}_{2}\left(\mathrm{Zr}_{\mathrm{y}} \mathrm{Ti}_{1-\mathrm{y}}\right)_{2} \mathrm{O}_{7}$ tanto la subred de aniones como la de cationes se desordenan gradualmente conforme se incrementa el contenido de $\mathrm{Zr}$, lo que conlleva a la generación 
de vacancias de oxígeno en la posición $48 f$ de la estructura, las cuales son conocidas por ser responsables del salto y la difusión de los iones oxígeno (22,23), lo que la hace ideal para llevar a cabo un estudio como el aquí presentado.

Los resultados obtenidos muestran que la transición al régimen de pérdidas dieléctricas constantes puede ser claramente identificada en estos materiales y que es un proceso térmicamente activado con una energía de activación $E_{N C L}$ mucho más baja que la energía de activación $E_{d c}$ para la conducción de largo alcance (24-26).

\section{DESARROLLO EXPERIMENTAL}

Las muestras estudiadas en este trabajo fueron sintetizadas mediante mecano-síntesis, partiendo de mezclas estequiométricas de óxidos elementales de alta pureza, utilizando un molino planetario Retsch PM 400 con dos estaciones de molienda, morteros de circonia estabilizada con itria de $125 \mathrm{ml}$ de capacidad y bolas de $2 \mathrm{~cm}$ de diámetro del mismo material. El proceso de síntesis está descrito ampliamente en publicaciones anteriores (26). La composición de los polvos extraídos del molino fue analizada mediante la técnica de difracción de rayos $\mathrm{X}$ en polvos en un difractómetro Phillips X'pert usando una radiación de $\mathrm{Cu} \mathrm{K \alpha}(\lambda=1.5418 \AA ̊)$. Las reacciones químicas se consideraron completas cuando no se observaron reflexiones características de los reactivos de partida mediante esta técnica. Las medidas de espectroscopia de impedancias fueron realizadas en pastillas cilíndricas de 7 $\mathrm{mm}$ de diámetro por $0.7 \mathrm{~mm}$ de espesor, obtenidas mediante prensado uniaxial y un posterior sinterizado a $1500^{\circ} \mathrm{C}$ de los polvos obtenidos por molienda, dando lugar a un tamaño de grano típicamente de 1-2 micras. Las dos caras opuestas de las pastillas fueron recubiertas con una capa de pintura de platino coloidal que actúa como electrodos bloqueantes. Las medidas se realizaron bajo un flujo de $\mathrm{N}_{2}$ y los datos de impedancia fueron registrados a frecuencias entre $1 \mathrm{~Hz}$ y $3 \mathrm{MHz}$ en función de la temperatura y con un voltaje aplicado ac de $0.5 \mathrm{~V}$, utilizando un analizador de impedancias Novocontrol de alta resolución.

\section{RESULTADOS Y DISCUSIÓN}

En la figura 1(a) se representa, en escala doble logarítmica, la dependencia de la parte real de la conductividad con la frecuencia, a diferentes temperaturas, correspondiente a la muestra de composición $\mathrm{Gd}_{2} \mathrm{Zr}_{2} \mathrm{O}_{7}$, la cual ha sido seleccionada como representativa. Se puede observar que a temperaturas suficientemente bajas o altas frecuencias, la conductividad $d c$ toma valores muy bajos y se hace presente el régimen de pérdidas dieléctricas constantes (NCL) y por consecuencia, la conductividad muestra una dependencia lineal con la frecuencia. Las líneas sólidas en la figura son ajustes de acuerdo a la ecuación $\sigma^{\prime}{ }_{N C L}(\omega)=A \omega \varepsilon_{0}$ que permiten identificar la frecuencia de transición a la cual el régimen del NCL finaliza a cada temperatura. El eje de las ordenadas en la figura se presenta en unidades arbitrarias ya que las isotermas correspondientes a $60,90,130$ y $190^{\circ} \mathrm{C}$ se han desplazado verticalmente 1, 2, 3 y 4 órdenes de magnitud respectivamente con la finalidad de mostrar de forma clara la región donde se presenta dicho régimen. A frecuencias menores, se observa una contribución adicional a la conductividad, que es consecuencia del salto local de los iones y que se evidencia en una dependencia potencial de la conductividad con la frecuencia, la cual eventualmente llega a ser independiente en el régimen de la conductividad $d c$, a frecuencias lo suficientemente bajas o altas temperaturas (ver datos de conductividad en $\mathrm{T}=190^{\circ} \mathrm{C}$ ). Con la finalidad de determinar de una forma más clara la frecuencia de transición al régimen NCL es conveniente representar los mismos datos mostrados en la figura 1(a) pero ahora para el valor de las pérdidas dieléctricas $\varepsilon^{\prime \prime}{ }_{N C L}(\omega)=A$, tal y como se observa en la figura 1 (b) y en donde se incluyen las frecuencia de transición (rombos). El criterio seleccionado para determinar a cada temperatura una frecuencia de transición $\left(\omega_{x}\right)$ en la cual el término NCL se hace la contribución dominante, fue independiente de cualquier modelo teórico, de tal manera que se definió dicha frecuencia cuando el valor de las pérdidas dieléctricas satisface la relación $\log \left[\varepsilon^{\prime \prime}\left(\omega_{x}\right) / \varepsilon^{\prime \prime}\left(10 \omega_{x}\right)\right]=0.05$, es decir que se desvía del valor casi constante cerca de un $10 \%$. Como se observa en la figura 1(b), el seleccionar este criterio permite una precisión suficiente para determinar la frecuencia $\omega_{x}$ ya que esta es muy cercana al rango de frecuencias donde el NCL se hace evidente. Es importante mencionar que al seleccionar diferentes criterios (desviaciones del 5 ó 20\%) se obtienen resultados similares a los aquí presentados con la única diferencia de que las frecuencias de transición son ligeramente diferentes a cada temperatura.

La dependencia de la conductividad ac con la temperatura, para la misma muestra $\left(\mathrm{Gd}_{2} \mathrm{Zr}_{2} \mathrm{O}_{7}\right)$ y en una representación de tipo Arrhenius, se presenta en la figura 2, a diferentes frecuencias fijas (isócronas) en el rango de $1 \mathrm{~Hz}-1 \mathrm{MHz}$ de abajo hacia arriba.

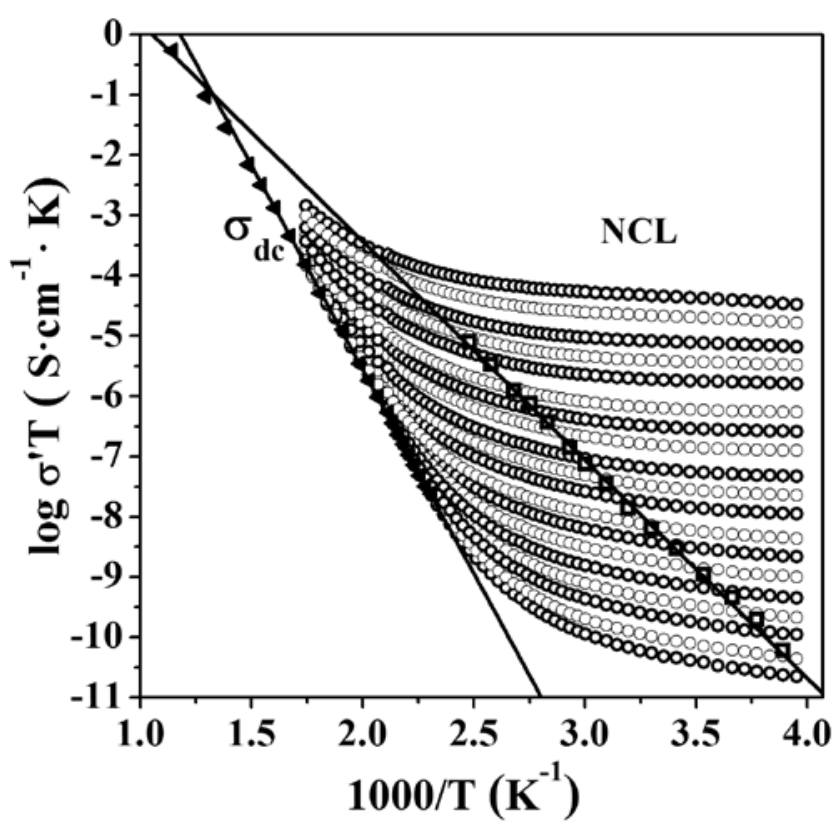

Figura 2. Representación de tipo Arrhenius, que muestra la dependencia de la parte real de la conductividad con la temperatura para el $\mathrm{Gd}_{2} \mathrm{Zr}_{2} \mathrm{O}_{7}$ a diferentes frecuencias fijas ( de $1 \mathrm{~Hz}$ a $1 \mathrm{MHz}$, tres frecuencias por década de abajo hacia arriba). Los puntos cuadrados corresponden a los valores de conductividad para la transición al régimen de pérdidas dieléctricas constantes (NCL). Los triángulos corresponden a los datos de la conductividad dc y las líneas sólidas son ajustes de mínimos cuadrados a los datos de acuerdo a una ley de tipo Arrhenius. 


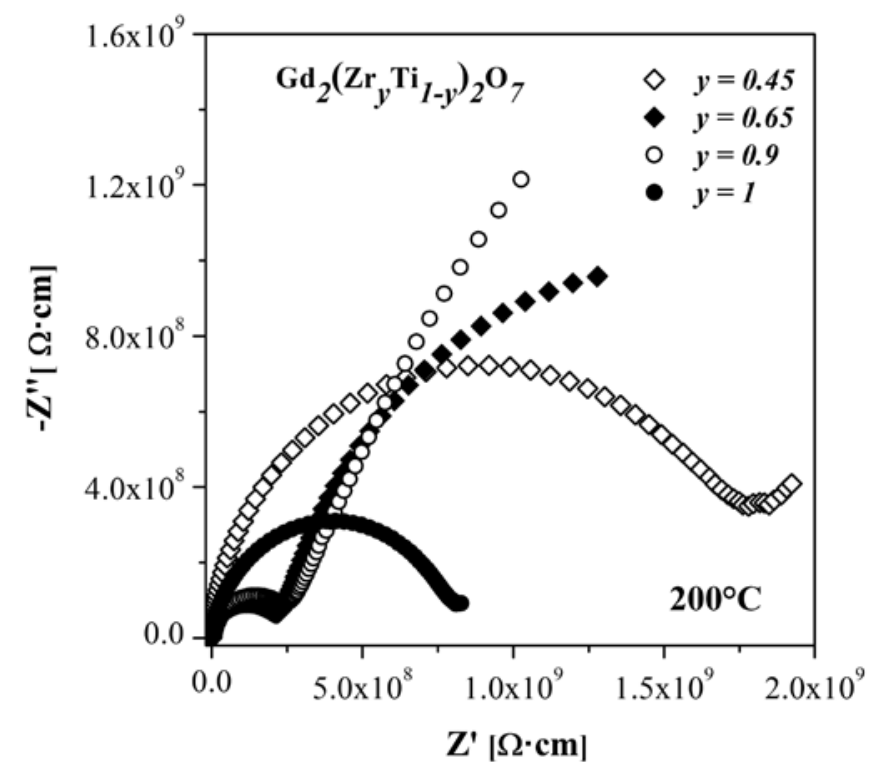

Figura 3. Gráfica de impedancia compleja (diagramas de Nyquist) para las distintas muestras estudiadas y a una misma temperatura, $200^{\circ} \mathrm{C}$. La frecuencia angular $(\omega)$ en las isotermas se incrementa de derecha a izquierda. Se puede apreciar que en este tipo de representación se hacen evidentes los semicírculos característicos encontrados en conductores iónicos policristalinos. El primero, un semicírculo incompleto, presente a frecuencias intermedias, corresponde a la contribución de la frontera de grano, mientras que el segundo que se observa a elevadas frecuencias corresponde a la contribución del volumen.

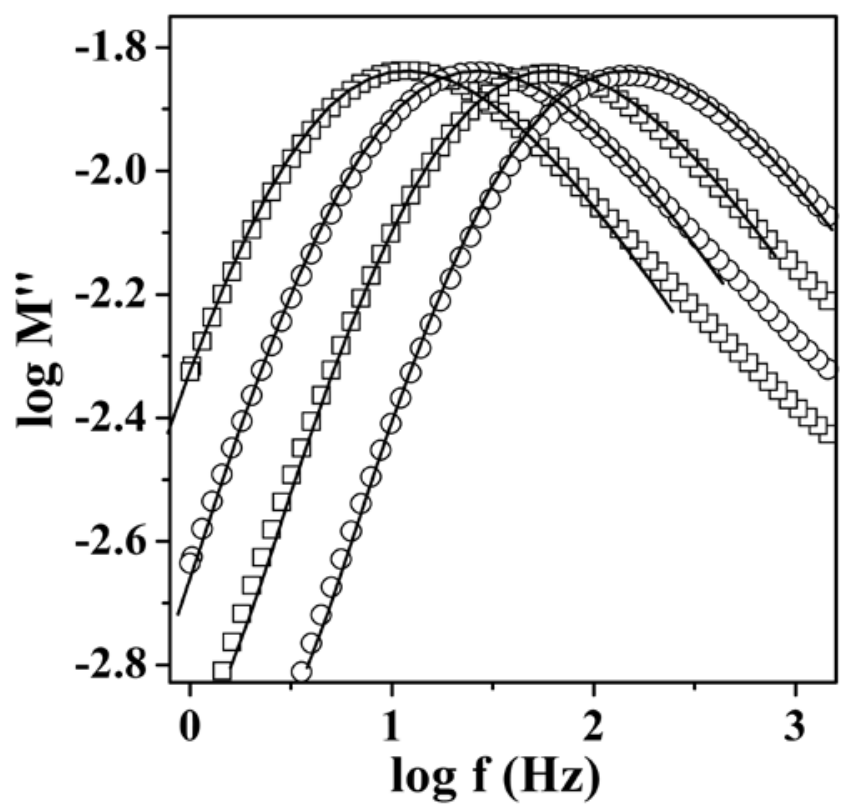

Figura 4. Dependencia de la parte imaginaria del módulo eléctrico en función de la frecuencia en escala doble logarítmica para el $\mathrm{Gd}_{2} \mathrm{Zr}_{2} \mathrm{O}_{7}$ a $\mathrm{T}=170,180,190$ y $200^{\circ} \mathrm{C}$ de izquierda a derecha. Las líneas sólidas representan ajustes de acuerdo a las ecuaciones (3) y (4).
Como se puede observar, los datos experimentales a elevadas temperaturas y a diferentes frecuencias tienden a colapsar en una sola curva como resultado del valor constante que toma la conductividad con la frecuencia, el cual corresponde a un régimen de conductividad $d c$ (triángulos), cuyo valor puede ser obtenido a partir de los diagramas de Nyquist para cada temperatura mediante el inverso del valor de la parte real de la impedancia $Z^{\prime}(\omega)$ a la frecuencia que separa la contribución del grano y de la frontera de grano (ver figura 3). Cuando se disminuye la temperatura, se entra en el régimen de salto iónico local, de tal forma que las isócronas se separan siguiendo una dependencia potencial en frecuencia. Finalmente, al disminuir aún más la temperatura, el régimen NCL se hace presente por lo que las isócronas están espaciadas de acuerdo a una dependencia lineal en frecuencia, la cual supone un valor casi constante en el valor de las pérdidas dieléctricas ( $\varepsilon^{\prime \prime}$. Es importante observar que el régimen NCL no solo se caracteriza por una dependencia lineal de la conductividad ac con la frecuencia, sino también por una dependencia muy suave con la temperatura y la cual usualmente se ha encontrado en conductores iónicos (4).

En la figura 2 se incluyen también, los valores de la conductividad de transición al régimen NCL (cuadrados), obtenidos de las isotermas de conductividad ac como se describió en la figura 1. De acuerdo a la literatura, en el caso de conductores de iones litio se encontró, que dicha transición en la conductividad estaba térmicamente activada, con una energía de activación $E_{N C L}=0.71 \pm 0.04$ $\mathrm{eV}$ mucho menor que la medida para la conductividad $d c$ $\left(E_{d c}=1.32 \pm 0.04 \mathrm{eV}\right)(11,28)$. Esto es de hecho, el resultado esperado para el NCL cuando los iones que se encuentran es su pozo de potencial, tienen una pequeña probabilidad de saltar y salir de dichos pozos a bajas temperaturas $(14,15)$. El valor de la energía de activación $E_{N C L}$ menor, podría corresponder a la energía asociada a la barrera de potencial que los iones oxígeno tienen que superar para saltar de un sitio a otro $E_{a^{\prime}}$ mientras que la energía de activación para el proceso de conducción de largo alcance $E_{d c}$ es mayor como consecuencia de una penalización energética que los iones han de superar en su movimiento y que es debida a la interacción mutua entre ellos o correlación. De acuerdo al modelo Coupling Model (CM) propuesto por Ngai es posible estimar la energía $E_{a}$ a partir de medidas de conductividad compleja realizadas a elevada temperatura, mediante el uso de la relación $(29,30)$ :

$$
E_{a}=(1-n) E_{d c}[2]
$$

donde $n$ es un exponente fraccionario comprendido entre 0 y 1 que caracteriza la función de correlación $\Phi(\mathrm{t})$ para el movimiento de los iones la cual se describe comúnmente en términos de una función del tipo Kohlrausch-Williams-Watts (KWW) de la forma (31):

$$
\Phi(\mathrm{t})=\exp \left(-(\mathrm{t} / \tau)^{1-\mathrm{n}}\right)
$$

Para poder determinar el valor del exponente $n$, a partir de datos experimentales, es necesario analizar la dependencia de la parte compleja del módulo eléctrico con la frecuencia $M^{*}(\omega)$, la cual se relaciona directamente con la conductividad compleja mediante la relación $\mathrm{M}^{*}(\omega)=j \omega \varepsilon_{0} / \sigma^{*}(\omega)$. Concretamente el módulo eléctrico se puede expresar en términos de la función 
$\Phi(\mathrm{t})$, por medio de la transformada de Fourier de una derivada temporal de la dicha función (32),

$$
M^{*}(\omega)=\frac{1}{\varepsilon_{\infty}}\left[1-\int_{0}^{\infty}\left(-\frac{\mathrm{d} \Phi}{\mathrm{dt}}\right) \mathrm{e}^{-\mathrm{j \omega t}} \mathrm{dt}\right]
$$

donde $\varepsilon_{\infty}$ es la permitividad dieléctrica para altas frecuencias. En la figura 4 se presenta la dependencia de la parte imaginaria $M^{\prime \prime}(\omega)$ del módulo eléctrico $M^{*}(\omega)$ en función de la frecuencia en una escala doble logarítmica, para la muestra de composición $\mathrm{Gd}_{2} \mathrm{Zr}_{2} \mathrm{O}_{7}$ seleccionada como representativa. Esta representación exhibe picos asimétricos como una respuesta del proceso de relajación de la conductividad iónica de este material. Se observa que el máximo ocurre a la frecuencia característica, $\omega_{p^{\prime}}$ que aumenta al incrementar la temperatura. Las líneas sólidas en la figura representan ajustes de acuerdo a la ecuaciones [3] y [4], aproximándose muy bien a los datos experimentales y a partir de los cuales se puede obtener el valor del exponente $n=0.44$, observándose que dicho valor es independiente de la temperatura para cada muestra. De esta manera, mediante el uso de la ecuación [2] y de los valores obtenidos de datos experimentales correspondientes a $n$ y $E_{d c}$ es posible determinar la barrera de potencial que tienen que superar los iones oxígeno para saltar de un sitio a otro, $E_{a}=0.74 \pm 0.04 \mathrm{eV}$, la cual es de hecho, muy cercana, tal y como se esperaba, al valor obtenido a partir de la figura 2 correspondiente a la energía $E_{N C L}=0.71 \pm 0.04 \mathrm{eV}$. Además, estos valores están de acuerdo con los obtenidos para este tipo de materiales mediante simulaciones (24). En este punto es

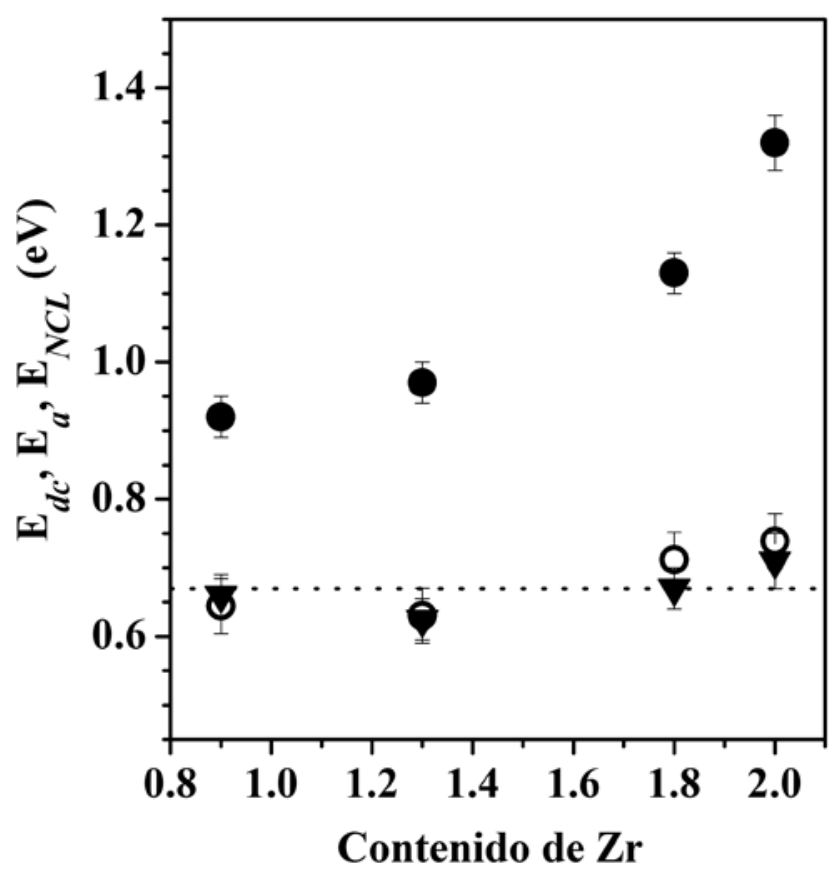

Figura 5. Dependencia de las energías de activación, $E_{d c}(\bullet)$ y $E_{a}(\mathrm{O}$ ) para el sistema $\mathrm{Gd}_{2}\left(\mathrm{Zr}_{\mathrm{y}} \mathrm{Ti}_{1-\mathrm{y}}\right)_{2} \mathrm{O}_{7}$ en función del contenido de $\mathrm{Zr}$. La línea punteada representa el valor promedio $E_{a}=0.67 \mathrm{eV}$ obtenido de la barrera de potencial para el salto de los iones oxígeno. La energía de activación $E_{N C L}(\mathbf{v})$ para la transición del régimen NCL también es graficada, mostrando valores similares a los de la barrera de potencial $E_{a}$ para todas las muestras. importante enfatizar que seleccionando un criterio diferente para determinar la frecuencia de transición (5 ó 20\% en lugar del $10 \%$ seleccionado) se obtienen los mismos valores para la energía de activación $E_{N C L^{\prime}}$ la cual es en consecuencia independiente de este criterio.

Recientemente se ha reportado que mediante la sustitución de $\mathrm{Ti}$ por $\mathrm{Zr}$ en el sistema $\mathrm{Gd}_{2}\left(\mathrm{Zr}_{\mathrm{y}} \mathrm{Ti}_{1-\mathrm{y}}\right)_{2} \mathrm{O}_{7}$ la energía de activación $E_{d c}$ se incrementa con el contenido de $\mathrm{Zr}$ a temperaturas superiores a $200^{\circ} \mathrm{C}(25,26)$. De manera interesante, se determinó que el valor de exponente $n$ que caracteriza la correspondiente función de correlación KWW también se incrementa con el contenido de Zr. Asimismo, el valor estimado para la barrera de potencial, $E_{a^{\prime}}$ obtenido de acuerdo a la ecuación [2], resultó ser independiente, dentro del error experimental, de la composición. Puesto que es bien conocido que el número de vacancias móviles de oxígeno en la posición $48 f$ se incrementa cerca de dos órdenes de magnitud cuando se incrementa el contenido de $\mathrm{Zr}$ de $y=0.3$ a $y=1$ en $\mathrm{Gd}_{2}\left(\mathrm{Zr}_{\mathrm{y}} \mathrm{Ti}_{1-\mathrm{y}}\right)_{2} \mathrm{O}_{7}(21,33)$, el incremento observado en $E_{d c}$ ha sido relacionado como una consecuencia del incremento en el valor del exponente $n$ debido a un aumento en los efectos de interacción o de correlación entre los iones oxígeno móviles, durante el proceso de conducción de largo alcance, a pesar de que $E_{a}$ no cambia significativamente $(25,26)$. De este modo, es también de esperar que el valor de la energía $E_{N C L}$ permanezca esencialmente independiente del contenido de $\mathrm{Zr}$ en el sistema $\mathrm{Gd}_{2}\left(\mathrm{Zr}_{\mathrm{y}} \mathrm{Ti}_{1-\mathrm{y}}\right)_{2} \mathrm{O}_{7}$ y que tome valores similares a los de $E$ para cada composición. Para poder comprobar esta predicción, se llevaron a cabo medidas y se realizó un análisis similar al presentado para el $\mathrm{Gd}_{2} \mathrm{Zr}_{2} \mathrm{O}_{7}$ pero, en este caso, para muestras con diferentes contenidos de Ti/Zr y los resultados correspondientes a la energía $E_{N C L}$ se presentan en la figura 5 . Se puede observar que dicha energía $\left(E_{N C L}\right)$ es independiente del contenido de $\mathrm{Zr}$, tal como se esperaba, y está de hecho, en excelente concordancia con los valores para la barrera potencial $E_{a}$ obtenidos a partir de la ecuación [2], mediante los valores experimentales $E_{d c}$ y $n$ para cada composición

\section{CONCLUSIÓN}

En resumen, el estudio del régimen de perdidas dieléctricas permitió determinar la frecuencia de transición a la cual éste régimen deja de hacerse presente en los datos experimentales obtenidos a partir de isotermas de conductividad ac para compuestos de fórmula general $\mathrm{Gd}_{2}\left(\mathrm{Zr}_{\mathrm{y}} \mathrm{Ti}_{1-\mathrm{y}}\right)_{2} \mathrm{O}_{7} \mathrm{y}$ con diferentes contenidos de Zr. Se encontró que dicha frecuencia esta térmicamente activada con una energía de activación similar a la energía de activación microscópica, $E_{a^{\prime}}$ que representa la barrera de potencial que los iones oxígeno móviles tienen que superar para saltar de una posición a otra. Esta última energía resultó ser significativamente menor que la energía de activación $E_{d c}$ que resulta del proceso de conducción de largo alcance de los iones oxígeno. Estos resultados ponen en evidencia la importancia de los efectos cooperativos o de interacción entre los iones móviles y la barrera de energía, en la determinación de los valores de la energía de activación $d c$ para el proceso de conducción e indican que el NCL en éste sistema es consecuencia de las vibraciones locales reflejadas en el desplazamiento cuadrático medio de los iones cuando estos permanecen confinados en su pozo de potencial. 


\section{AGRADECIMIENTOS}

La presente investigación ha sido financiada mediante los proyectos: CONACYT (SEP-2003-CO2-44075), MICINN (MAT2008-6517-CO2), CONSOLIDER INGENIO 2010 (CSD2009-00013), PHAMA CAM (S2009/MAT-1756). M.R. Díaz-Guillén agradece al Conacyt por la beca posdoctoral otorgada.

\section{BIBLIOGRAFÍA}

[1] W.K. Lee, J.F. Liu, A.S. Nowick, "Limiting behavior of ac conductivity in ionically conducting crystals and glasses: A new universality", Phys. Rev. Lett. 67, 1559-1561 (1991).

[2] J. Wong and C.A Angell, Glass structure by spectroscopy (Dekker, New York, 1976).

[3] C. H. Hsieh and H. Jain, "Are the low temperature-low frequency and high temperature-high frequency ac conductivity of glasses the same phenomenon?", J. Non-Cryst. Solids 203, 293-299 (1996).

[4] K.L. Ngai, "Properties of the constant loss in ionically conducting glasses, melts, and crystals", J. Chem. Phys. 110, 10576-10584 (1999).

[5] C. Cramer, K. Funke and T. Saatkamp, "Ion dynamics in glass-forming systems: I. Conductivity spectra below the glass transformation temperature", Phil. Mag. B 71, 701 (1995).

[6] D. L. Sidebottom, P. F. Green and R. K. Brow, "Two Contributions to the ac Conductivity of Alkali Oxide Glasses", Phys. Rev. Lett. 74, 5068 (1995).

[7] A. S. Nowick, A. V. Vaysleb and W. Liu, "Identification of distinctive regimes of behaviour in the ac electrical response of glasses", Solid State Ionics 105, 121-128 (1998).

[8] H. Jain and S. Krishnaswami, "Composition dependence of frequency power law of ionic conductivity of glasses" Solid State Ionics 105, 129-137 (1998).

[9] C. Leon, M. L. Lucía, J. Santamaria, F. Sánchez-Quesada, “Universal scaling of the conductivity relaxation in crystalline ionic conductors", Phys. Rev. B 57, 41-44 (1998).

[10] C. Leon, K. L. Ngai, and A. Rivera, "Correlation between ion hopping conductivity and near constant loss in ionic conductors", Phys. Rev. B 69, 134303 (2004).

[11] C. Leon, A. Rivera, A. Várez, J. Sanz, J. Santamaria and K. L. Ngai, “Origin of constant loss in ionic conductors", Phys. Rev. Lett. 86, 1279-1282 (2001).

[12] A. Rivera, C. Leon, C. P. E. Varsamis, G. D. Chryssikos, K. L. Ngai, C. M. Roland, and J. L. Buckley, "Cation mass dependence of the nearly constant dielectric loss in alkali triborate glasses", Phys. Rev. Lett. 88, 125902 (2002).

[13] A. Rivera, C. Leon, J. Sanz, J. Santamaria, C. T. Moynihan and K. L. Ngai, "Crossover from ionic hopping to nearly constant loss in the fast ionic conductor $\mathrm{Li}_{0.18} \mathrm{La}_{0.61} \mathrm{TiO}_{3}$ ", Phys. Rev. B. 65, 224302 (2002).

[14] K. L. Ngai and C. Leon, "Cage decay, near constant loss, and crossover to cooperative ion motion in ionic conductors: Insight from experimental data", Phys. Rev. B 66, 064308 (2002).

[15] K. Funke, P. Singh and R. D. Banhatti, "Conductivity dispersion in supercooled calcium potassium nitrate: caged ionic motion viewed as part of standard behavior", Phys. Chem. Chem. Phys. 9, 5582-5590 (2007).

[16] J. Ross Macdonald, "Some alternate models for nearly constant loss in conductive systems", Phys. Rev. B 66, 064305 (2002).
[17] W. Dieterich and P. Maass, "Some alternate models for nearly constant loss in conductive systems", Chem. Phys. 284, 439-467 (2002).

[18] B. Roling, C. Martiny, and S. Murugavel, "Ionic conduction in glass: New Information on the interrelation between the 'Jonscher behavior' and the 'Nearly constant-loss behavior' from broadband conductivity spectra", Phys. Rev. Lett. 87, 085901 (2001).

[19] J. Habasaki, K. L. Ngai, and Y. Hiwatari, "Molecular dynamics study of cage decay, near constant loss, and crossover to cooperative ion hopping in lithium metasilicate", Phys. Rev. E 66, 021205 (2002).

[20] D. M. Laughman, R. D. Banhatti and K. Funke, "New nearly constant loss feature detected in glass at low temperatures", Phys. Chem. Chem. Phys. 11, 3158-3167 (2009).

[21] P. K. Moon and H. L. Tuller, " Ionic Conduction in the $\mathrm{Gd}_{2} \mathrm{Ti}_{2} \mathrm{O}_{7}-\mathrm{Gd}_{2} \mathrm{Zr}_{2} \mathrm{O}_{7}$ system", Solid State Ionics 28-30, 470-474 (1988).

[22] J. Chen, J. Lian, L. M. Wang, R. C. Ewing, R. G. Wang, and W. Pan, " $X$-ray photoelectron spectroscopy study of disordering in $\mathrm{Gd}_{2} \mathrm{Zr}_{2-\mathrm{y}} \mathrm{Ti}_{\mathrm{y}} \mathrm{O}_{7}$ pyrochlores", Phys. Rev. Lett. 88, 105901 (2002).

[23] P. K. Moon and H. L. Tuller, in Solid State Ionics, edited by G. Nazri, R. A. Huggins, and D. F. Shriver MRS Symposia Proceedings No. 135 (Materials Research Society, Pittsburgh), 149 (1989).

[24] R.E. Williford, W.J. Weber, R. Devanathan, J.D. Gale, "Effects of cation disorder on oxygen vacancy migration in $\mathrm{Gd}_{2} \mathrm{Ti}_{2} \mathrm{O}_{7}$ ", J. Electroceram. 3-4, 409-424 (1999).

[25] K. J. Moreno, G. Mendoza-Suárez, A. F. Fuentes, J. Garcia-Barriocanal, C. Leon and J. Santamaria, "Cooperative oxygen ion dynamics in $\mathrm{Gd}_{2} \mathrm{Ti}_{2}$ $\mathrm{Zr}_{y} \mathrm{O}_{7}$ ", Phys. Rev. B 71, 132301 (2005)

[26] M. R. Díaz-Guillén, K. J. Moreno, J. A. Díaz-Guillén, A. F. Fuentes, K. L. Ngai, J. Garcia-Barriocanal, J. Santamaria and C. Leon, "Cation size effects in oxygen ion dynamics of highly disordered pyrochlore-type ionic conductors", Phys. Rev. B 78, 104304 (2008).

[27] K. L. Ngai, G. N. Greaves, and C. T. Moynihan, “Correlation between the activation energies for ionic conductivity for short and long time scales and the Kohlrausch stretching parameter $\beta$ for ionically conducting solids and melts", Phys. Rev. Lett. 80, 1018-1021 (1998).

[28] Un valor de $E_{\mathrm{dc}}=1.13 \mathrm{eV}$ fue reportado en la referencia 26. La diferencia con el valor aquí reportado es debido al distinto rango de temperaturas utilizado en ambos trabajos para calcularla, puesto que hay una ligera y gradual disminución de dicha energía cuando la temperatura se incrementa por encima de $300^{\circ} \mathrm{C}$. Esta propiedad ha sido previamente reportada en la referencia 27 como característica universal de los conductores iónicos.

[29] K. L. Ngai, "Universality of low-frequency fluctuation, dissipation and relaxation properties of condensed matter", Comments Solid State Phys. 9, 121 (1979)

[30] K. Y. Tsang and K. L. Ngai, "Dynamics of relaxing systems subjected to nonlinear interactions", Phys. Rev. E 56, R17 (1997).

[31] C. T. Moynihan, "Description and analysis of electrical relaxation data for ionically conducting glasses and melts", Solid State Ionics 105, 175-183 (1998)

[32] K. L. Ngai, C. Leon, "Relating macroscopic electrical relaxation to microscopic movements of the ions in ionically conducting materials by theory and experiment", Phys. Rev. B 60, 9396-9405 (1999).

[33] C. Heremans, B. J. Wuensch, J. K. Stalick, and E. Prince, “Fast ion-conducting $\mathrm{Y}_{2}\left(\mathrm{Zr}_{\mathrm{Ti}} \mathrm{O}_{7}\right)$ Pyrochlores: Neutron Rietveld Analysis of Disorder Induced by Zr Substitution", J. Solid State Chem. 117, 108-121 (1995).

Recibido: $13 / 06 / 2011$

Aceptado: 28/10/2011 